\title{
LA POBREZA DE LAS FUENTES EN LA COBERTURA DE SUCESOS DE IMPACTO POR LOS DIARIOS DE REFERENCIA EN ESPAÑA ${ }^{1}$
}

\author{
Iñigo Marauri Castillo \\ (Universidad del País Vasco) \\ inigo.marauri@ehu.es \\ María del Mar Rodríguez González \\ (Universidad del País Vasco) \\ mirenr@gmail.com \\ María José Cantalapiedra González \\ (Universidad del País Vasco) \\ mariajose.cantalapiedra@ehu.es
}

\begin{abstract}
Resumen: Uno de los indicadores fundamentales para la medición de la calidad de un diario se halla en las fuentes informativas que utiliza, tanto en el aspecto cuantitativo como en el cualitativo. El presente artículo analiza ambos parámetros en la cobertura de los sucesos de impacto (hechos trágicos que generan conmoción social) de los principales diarios de referencia en España (El País, El Mundo, Abc, La Vanguardia, El Periódico de Catalunya, El Correo y Diario 16) entre 1977 y 2000. Para ello, se ha establecido una comparación entre las cabeceras y se han observado su posible evolución y las tendencias más relevantes.
\end{abstract}

Palabras Clave: Sucesos, crímenes, accidentes, fuentes, periódicos

Abstract: One of the key indicators for measuring the quality of a journal is in the information sources used, both quantitatively and qualitatively. This article analyzes both parameters in the coverage of accident and crime reports of the leading reference newspapers in Spain (El País, El Mundo, ABC, La Vanguardia, El Periódico de Catalunya, El Correo and Diario 16) between 1977 and 2000. To do so, this article includes a comparison among the various papers and the research of the evolution and possible trends.

Keywords: Accident and crime reports, sources, newspapers

${ }^{1}$ Este trabajo se ha realizado con la ayuda económica del programa de formación de investigadores del Gobierno. 
82 La pobreza de las fuentes en la cobertura de sucesos de impacto por los diarios de referencia en España

\section{INTRODUCCIÓN}

a cantidad y la calidad de las fuentes que utilizan, y que citan, los periodistas en su labor informativa suponen uno de los motivos y una de las preocupaciones más relevantes entre los investigadores del periodismo. Lo son porque las fuentes, entendidas como "el conjunto de elementos materiales o personales consultados por un autor al objeto de documentarse y servirse de los contenidos obtenidos en las mismas para la configuración de las unidades redaccionales" (Chimeno, 1997: 45) representan uno de los pilares de la labor periodística. Su relevancia es capital, además, en el periodismo impreso. Uno de los argumentos utilizados para justificar el mayor peso periodístico de los diarios de referencia frente a los medios de comunicación audiovisuales y digitales radica precisamente en la variedad y en la riqueza de sus fuentes.

Sin embargo, gana terreno la idea de que para poder competir con la radio, la televisión e Internet, la prensa de referencia se halla en pleno proceso de abandono de las señas de identidad que la caracterizaban, como eran el pluralismo competitivo que diferenciaba a las cabeceras, la estricta separación entre información (news) y opinión (views), el contraste de la noticia a través de múltiples fuentes y el estricto respeto de la intimidad personal, a la que nunca se invadía sin necesidad (Gil Calvo, 2002: 16).

De cualquier modo, se ha generalizado la percepción de pérdida en el contacto, alimentación y utilización de fuentes periodísticas en la cobertura informativa de los periódicos de referencia en España. La relevancia de las fuentes, de su presencia o de su ausencia, se acrecienta en el trabajo periodístico relacionado con los sucesos, un ámbito de la actualidad tan demandado por los lectores como delicado para los redactores.

\section{OBJETIVOS E HIPÓTESIS}

Los objetivos que han guiado la investigación recogida en este artículo son:

1. Conocer el alcance de las investigaciones sobre fuentes informativas realizado en los últimos años en España.

2. Comprobar el uso cuantitativo y cualitativo de las fuentes en los sucesos de gran impacto mediático dentro de los diarios de referencia en España.

3. Prestar especial atención al uso de testigos y de familiares de víctimas de sucesos como fuente informativa en los periódicos de referencia.

4. Analizar la tendencia registrada en el periodo estudiado (1977-2000) y las diferencias entre los distintos diarios analizados.

\footnotetext{
${ }^{65}$ Ante la imposibilidad de revisar ejemplar a ejemplar los 24 años señalados como periodo de estudio, se ha optado por utilizar como fuente los índices anuales de artículos de algunos de los diarios analizados.

${ }^{66}$ Datos obtenidos en VV.AA (2002). Libro blanco de la prensa diaria. AEDE. Madrid. Pp. 56-57.
} 
El cumplimiento de estos objetivos busca comprobar las hipótesis que se desglosan a continuación:

1. Desde el punto de vista cuantitativo, el uso de las fuentes en las informaciones sobre sucesos de relevancia en los diarios de referencia en España es insuficiente. Abundan las informaciones sin atribución de fuente, y escasean las informaciones que cuentan con más de dos fuentes explícitas.

2. En una perspectiva cualitativa, la aportación de las fuentes depende en exceso de las instituciones, cuya información no siempre se contrasta.

3. Es habitual la utilización de declaraciones de testigos.

4. Se utiliza con exceso y de forma poco cuidadosa las declaraciones de familiares de las víctimas de los sucesos.

5. Desde una perspectiva diacrónica, las deficiencias en el uso de las fuentes no se han resuelto con el paso de los años.

6. Las diferencias en el tratamiento y uso de las fuentes entre los diarios analizados son notables.

Se puede establecer comunicación a través de la simple transmisión del mensaje más común (la palabra), pero es posible también con.

seguir una comunicación más fácil de percibir por el receptor, más comprensible y más rica en matices expresivos a través de la creación artística. Para hacer del lenguaje radiofónico una obra de arte que permita crear imágenes más precisas e impactantes en la mente del destinatario, es necesario elegir, combinar y emitir los elementos sonoros de la manera más adecuada posible. El oyente percibe un determinado sonido y realiza inmediatamente en su cerebro una asociación de este sonido con una imagen, un sentimiento o una sensación. Cuando escucha las retransmisiones de los partidos de fútbol de la jornada, el oyente recrea en su mente lo que está sucediendo en el terreno de juego a partir de lo que le transmite el narrador a través de las ondas.

\section{ESTADO DE LA CUESTIÓN}

El de las fuentes es uno de los campos de mejora del periodismo de sucesos y tribunales. Así lo atestiguan los escasos autores que lo han abordado en España. La investigadora Carmen Herrero establece un exhaustivo listado de "los problemas más habituales y graves" en la cobertura informativa de los sucesos, en el que incluye el recurso a fuentes anónimas y poco fiables o la excesiva dependencia de fuentes oficiales; el uso de rumores y conjeturas o la utilización de datos de la vida privada de las personas implicadas en los hechos narrados (Herrero, 2003: 87).

Junto con Carmen Herrero, otra de las excepciones dentro de la escasez de 
84 La pobreza de las fuentes en la cobertura de sucesos de impacto por los diarios de referencia en España

monografías y manuales sobre el trabajo de los periodistas que cubren crímenes, catástrofes o accidentes en el mundo hispano se encuentra en el libro Periodismo de sucesos, obra de la catedrática de la Universidad Pompeu Fabra Montse Quesada, que ya en su introducción recalca que "cada vez es más necesario y urgente que el Periodismo de sucesos sea abordado desde la especialización, es decir, desde los procesos de producción de la información especializada" (Quesada, 2007: 14).

Las investigadoras Eleanor Singer y Phillys M. Endreny apuntan en su trabajo Reporting on risk que la cobertura de desastres naturales y accidentes se centra en el drama y el conflicto más que en una visión a largo plazo, con un tratamiento superficial y fragmentado de la realidad, tratamiento que está condicionado de forma particular por la dependencia de las fuentes institucionales y los intereses de la audiencia (Singer y Endreny, 1993: 41).

Ambas influencias, las de las fuentes institucionales y las de los intereses de la audiencia, condicionan el periodismo de las últimas tres décadas. Ya lo advierte el catedrático de la Universidad de La Laguna José Manuel De Pablos cuando señala que se tiende hacia un periodismo más ligero, con unos medios cuya elaboración resulta más barata, gracias en gran medida a la avalancha de información precocinada en otros organismos (gabinetes de comunicación principalmente), avalancha que provoca que los redactores salgan menos a la calle (De Pablos, 2006: 119). En palabras de Ofa Bezunartea (Bezunartea, Del Hoyo y Martínez, 1998: 85), la excesiva utilización de las fuentes oficiales es un problema universal que se asocia a la "funcionarización" de los redactores, materializada en la aplicación de fórmulas rutinarias en el trabajo periodístico que se basan en la dependencia de fuentes oficiales u oficializadas.

Se ha producido una perversa inversión de papeles. Los periodistas no buscan fuentes; las fuentes, especialmente las fuentes profesionalizadas, buscan a los periodistas en sus redacciones. Lo explica gráficamente Herbert Gans cuando describe las relaciones entre fuentes y periodistas como una danza, como un tango concretamente, en el que unos u otros pueden dirigir el baile. Sin embargo, y como él mismo señala, son las fuentes quienes lo hacen con mayor frecuencia (Gans, 1980: 116).

Un estudio del empleo de las fuentes en los diarios de información general de Galicia constata (López y García, 2005) que los periodistas abusan de las fuentes oficiales, ocultan la identidad de las fuentes sin causas suficientemente justificadas y aceptan la mayoría de las condiciones que establecen las fuentes para la elaboración y difusión de la información (López, 2006: 39).

Estos profesionales de la información carecen, en muchos casos, de agenda propia, y su red de contactos se circunscribe a los gabinetes de comunicación, a los que les une una peligrosa relación de dependencia. Las consecuencias nocivas de esta debilidad, como recoge José Vicente García Santamaría en su análisis sobre la cobertura de la tragedia aérea ocurrida en el aeropuerto de 
Barajas en 2008 (García Santamaría, 2010), se agudizan en el periodismo de sucesos, en el que la información oficial siempre es escueta, mínima y muchas de las informaciones posteriores se proporcionan casi siempre por goteo, cuando no son el fruto de filtraciones dirigidas. De ahí la obligación de todo buen periodista de sucesos de ir siempre más allá de lo que pueden ofrecerle las fuentes oficiales (Quesada, 2007: 19).

Y lo cierto es que la fuente es esencial en la actividad informativa y marca, como vértice central de la actividad periodística, el sello de distinción de los medios de comunicación (Armentia y Caminos, 2002). Su presencia no sólo no se puede soslayar en el ciclo informativo (Borrat, 2003:76), sino que representa un elemento fundamental en la construcción del relato periodístico. La fuente es el punto de partida de todo proceso comunicativo, pero, como bien señala Chimeno, su utilización exige criterios de actuación profesional y de control (Chimeno, 1997: 46).

\section{METODOLOGÍA}

\subsection{Corpus del estudio}

La investigación se ha basado en dos pilares. Por un lado, un exhaustivo trabajo de documentación para conocer el estado de la cuestión. Por otro, el estudio detallado de los datos obtenidos a través del análisis de contenido, materializado en una disección cuantitativa y también cualitativa de una muestra representativa de la cobertura informativa de los sucesos en una selección de los principales diarios de información general españoles entre 1977 y 2000 . La elección de este periodo no responde a un motivo casual o arbitrario. Tras la muerte del dictador Francisco Franco el 20 de noviembre de 1975, se abre una nueva etapa política y social en España que se materializará en la llegada de la democracia y, con ella, de la libertad de prensa. La ausencia de este derecho durante el franquismo no sólo afectó a parcelas de la información relacionadas con los grandes asuntos de la vida política, económica y social. También los sucesos se vieron cercenados por la censura, al menos durante la primera etapa de la dictadura. Como recuerda Amparo Moreno Sardá (1998: 118), la censura franquista dosificó el número de homicidios que podían publicarse en cada número de las revistas de sucesos, aunque esta práctica disminuyó desde mediados de los años cincuenta.

En el proceso democrático español, y gracias a él, los medios de comunicación en general y la prensa en particular viven un cambio y unas transformaciones históricas. Los historiadores Juan Francisco Fuentes y Javier Fernández Sebastián resaltan que con el reconocimiento por la Constitución de 1978 del derecho "a expresar y difundir libremente los pensamientos, ideas y opiniones mediante la palabra, el escrito o cualquier otro medio de reproducción", así como "a comunicar o recibir información veraz por cualquier medio de difusión" (artículo 20.1 a y 20.1.d), la prensa española conseguía el marco jurídico necesario para desarrollar 
86 La pobreza de las fuentes en la cobertura de sucesos de impacto por los diarios de referencia en España

su función sin otras limitaciones que las que establecía el artículo 20.4 de la propia Constitución: "el derecho al honor, a la intimidad, a la propia imagen y a la protección de la juventud y de la infancia" (1998: 328). A la vista de estos hechos, el carácter crucial de este periodo apoya la idoneidad de la ubicación temporal de los diarios analizados.

A ello se suma la influencia que la transición ejerce sobre el tratamiento de los sucesos, como destaca Montse Quesada. Una de las primeras reformas significativas que propició la incipiente transición democrática en el Periodismo de sucesos fue el cambio de perspectiva informativa. Si hasta entonces el tema principal sobre el que se centraba la máxima atención periodística era sobre el propio hecho criminal, a partir de la transición democrática se empezó a ofrecer, además de esa información básica sobre el suceso, el contexto social y cultural en el que vivían los presuntos autores y las víctimas afectadas por el hecho criminal (2007: 35).

La elección del año 2000 como fin del periodo analizado tampoco es casual. La pujanza de Internet como medio de información se intensifica en los primeros años del siglo XXI, y uno de los principales afectados es precisamente la prensa diaria de referencia. Pese a que los datos obtenidos en las encuestas del Estudio General de Medios apuntan a un crecimiento del consumo de periódicos en papel entre 2000 y 2007 en España, este fenómeno solo se explica por la irrupción de la prensa gratuita (Larrañaga, 2009) con unas características que poco o nada tienen que ver con las principales cabeceras del país. De hecho, otros autores inciden en que España, aquejada ya de por sí de una de las más bajas tasas de lectura de diarios de Europa, no es ajena a la caída de difusión de las cabeceras de prensa registrada en la Unión Europea entre 1997 y 2007 (Armentia y Marín, 2009).

Se ha optado por acotar el campo de análisis a una serie de sucesos que comparten unos elementos determinados que reducen el ámbito de estudio de manera considerable sin anular ni el interés ni la validez científica del trabajo, tal y como aconseja Albert Kientz (Kientz, 1974: 166).

Por un lado, habida cuenta de la relevancia que atesoran en cualquier información, y en los sucesos más si cabe, los factores de proximidad geográfica y social, se ha delimitado el campo de estudio a los hechos que se han producido en territorio español o que han afectado a ciudadanos de esta nacionalidad.

Por otro, se han desechado los sucesos sin víctimas mortales. Es en la cobertura de la muerte por accidente, crimen o catástrofe cuando se pueden apreciar en mayor medida y de manera más clara las principales características de la plasmación de estos acontecimientos.

El tercer filtro se centra en el impacto generado. Se han establecido como requisitos la existencia de al menos un editorial o, si no es así, la aparición durante tres o más días de informaciones relacionadas con el suceso. 
Se ha procedido a un registro exhaustivo del periodo estudiado para conocer con exactitud el número de sucesos que cumplen las tres condiciones ${ }^{65}$.

En total, 107 noticias encajan dentro de los criterios marcados. De estas, se han seleccionado once, un $10 \%$ del conjunto, con dos criterios: temático y diacrónico. Temático, con el fin de que queden recogidos los principales tipos de suceso: catástrofes naturales, crímenes y accidentes aéreos y de otros medios de transporte colectivos. Diacrónico, para observar los posibles cambios y tendencias a lo largo del periodo estudiado, tanto entre los sucesos objeto de estudio como entre los diarios que componen la muestra.

Los sucesos seleccionados son los siguientes:

1. Accidente aéreo en el aeropuerto de Los Rodeos (1977).

2. Explosión de un camión cisterna junto al camping de Los Alfaques (1978).

3. Explosión de gas en un colegio de Ortuella (1980).

4. Incendio de la discoteca Alcalá 20 (1983).

5. Accidente aéreo en el monte Oiz (1985).

6. Choque de un tren con un autobús escolar en Juneda (1988).

7. Los crímenes de Puerto Hurraco (1990).

8. Los crímenes de Alcàsser (1992).

9. El crimen del Rol (1994).

10. La riada de Biescas (1996).

11. Accidente de un autobús de escolares en Golmayo (2000).

Perfilado ya el listado de sucesos cuya cobertura informativa será objeto de análisis, el siguiente paso viene dado por la necesidad de conocer qué diarios y cuántos ejemplares de cada uno de ellos se incluirán en el trabajo de campo. De los 84 diarios de información general con difusión registrada que circulaban en España entre 1990 y $2000^{66}$, se han seleccionado los siguientes: El País, Abc, La Vanguardia de Barcelona, El Periódico de Catalunya, El Correo Español-El Pueblo Vasco, El Mundo del siglo XXI y Diario 16. Los criterios que han guiado la elección de estas cabeceras han sido, en esencia, dos. Por un lado, la alta difusión de todos ellos en el periodo estudiado. Por otro lado, los siete diarios se han erigido durante el periodo de investigación como los principales referentes 
88 La pobreza de las fuentes en la cobertura de sucesos de impacto por los diarios de referencia en España

de la prensa diaria de información general en España.

Su modo de cubrir las noticias, en el aspecto formal y en el plano del contenido, marcan, de una forma u otra, la tendencia a seguir por el resto de diarios españoles. Además, sus particulares enfoques y diseños permiten que el investigador disponga en su estudio de los grandes referentes en la prensa española. En el caso de Diario 16, se ha limitado su presencia en la investigación hasta el año 1989, fecha en la que surge El Mundo del Siglo XXI. Éste último nace como alternativa de parte del hasta entonces equipo directivo de Diario 16 , al que los dueños de la empresa editora habían despedido meses antes, y de una notable representación de los redactores. Su estilo comparte, por tanto, muchas similitudes con Diario $16 \mathrm{y}$, de hecho, se produce una traslación en masa de los lectores de uno a otro. Fuentes y Fernández Sebastián (1998: 333) señalan que habría que catalogar a El Mundo, más que como un periódico de nueva creación, como un continuador del estilo periodístico y de la línea informativa de Diario 16 en la larga etapa que estuvo dirigido por Pedro J. Ramírez. Por todo ello, se ha considerado más adecuado la sustitución a partir de esa fecha de Diario 16 por El Mundo del Siglo XXI.

Queda por definir el número de ejemplares de cada diario que requiere el trabajo de campo. Con el fin de fijar una regla común para todos los casos que sirva como referencia, se ha optado por examinar la cobertura informativa de cada suceso publicada en un plazo de diez días desde la aparición en cada uno de los periódicos de la primera referencia vinculada con el suceso, periodo que permite analizar de forma idónea una cantidad de información muy amplia, pero abarcable y representativa. El número de unidades redaccionales que encajan en estos criterios de acotación y que se han convertido en el cuerpo de la investigación asciende a 1.750 .

\subsection{Categorización}

El análisis que sustenta el estudio se basa en el volumen de información recopilado por medio de una ficha de categorización estandarizada diseñada ex profeso para esta investigación. Para la agrupación y combinación del volumen de datos obtenidos se ha optado por utilizar como elementos estructurales los once grandes sucesos y los siete diarios seleccionados. Esta clasificación ofrece una doble perspectiva: por un lado, una visión de conjunto de las transformaciones generales y, por otro, un detallado informe de las diferencias entre cada uno de los diarios objeto de estudio.

\subsubsection{Categorías y variables}

Las categorías son las siguientes: número de suceso, número de texto (en adelante identificado como unidad redaccional), diario, fecha, fuentes principales, número de fuentes, declaraciones de testigos y declaraciones de afectados y 
de familiares de los afectados. Se detalla a continuación las variables de estas cuatro últimas categorías.

A) Fuentes principales: Se han fijado once variables y se ha utilizado como referencia para ello la clasificación establecida por Coca y Diezhandino (1997: 54).

1. Institucional, cuando las fuentes son o pertenecen a instituciones públicas o privadas definidas e identificables y hablan en representación o en calidad de ambas.

2. No institucional, cuando las fuentes no provienen de institución o de representante de institución alguna o, si lo son, hablan a título personal.

3. Ambas, cuando parte de las fuentes principales responden a la primera variable y parte a la segunda.

4. Observación directa, cuando la información proviene del testimonio personal sobre el suceso del propio redactor.

5. No consta, cuando en la unidad redaccional no se citan fuentes o cuando "se utilizan expresiones lo suficientemente ambiguas como para que no sea posible una determinación exacta, tales como fuentes de la Administración, fuentes próximas a... o fuentes fiables".

6. Diarios, cuando se citan textos publicados en otros periódicos o medios escritos.

7. Agencias, cuando la fuente principal es el texto elaborado por una agencia informativa.

8. Institucional, no institucional y observación directa, cuando se produce una combinación de las tres.

9. Observación directa y no institucional, para los casos en los que predominen estos dos tipos de fuentes.

10. Observación directa e institucional, por la misma razón.

11. Otras, para aquellas que no se puedan englobar en ninguna de las anteriores.

B) Número de fuentes: En esta categoría se establecen cuatro variables:

1. Una fuente, cuando en la unidad se utiliza una única fuente.

2. Dos fuentes, en el caso de que usen dos fuentes informativas.

3. Tres o más fuentes, para las unidades en las que aparecen tres o más fuentes informativas.

4. Valor cero, cuando no aparece fuente alguna en la unidad redaccional. 
90 La pobreza de las fuentes en la cobertura de sucesos de impacto por los diarios de referencia en España

C) Declaraciones de testigos: Se considera testigos a las personas que han presenciado el hecho relatado y no se han visto afectadas por sus consecuencias. Las variables son cuatro:

1. La unidad no cuenta con declaraciones de testigos.

2. Cuenta con declaraciones de un testigo.

3. Cuenta con declaraciones de dos testigos.

4. Cuenta con declaraciones de tres o más testigos.

D) Declaraciones de implicados o de familiares de los implicados: Esta categoría registra el uso de declaraciones de implicados en el suceso, bien como víctimas o como victimarios, o de sus familiares como fuente. Dispone de cuatro variables:

1. No cuenta con declaraciones de familiares o implicados.

2. Cuenta con una declaración de un familiar o un implicado.

3. Cuenta con declaraciones de dos familiares o implicados.

4. Cuenta con declaraciones de tres o más familiares o implicados.

\section{ANÁLISIS}

\subsection{Origen de las fuentes}

A tenor de los datos obtenidos, se constata que en una de cada tres unidades redaccionales analizadas la fuente de obtención de información sobre el suceso se limita a instituciones públicas (gobiernos nacional, autonómico o local, organizaciones de rescate y ayuda como la Cruz Roja o Protección Civil) o privadas (empresas afectadas en los hechos). De las 1.750 unidades estudiadas, en 606 la fuente es institucional, esto es, casi un 35\% del total. Este dato está en sintonía con lo que advierten Herrero y García Gordillo, cuando señalan que el periodista suele establecer una jerarquización de las fuentes que, en general, privilegia a la fuente oficial (Herrero y García Gordillo, 2003: 254).

Este amplio porcentaje contrasta con la afirmación de Montse Quesada, que defiende que la "obligación" de todo buen periodista de sucesos es ir siempre más allá de lo que puedan ofrecerle las fuentes oficiales de información y la necesidad de contar con un amplio abanico de fuentes especializadas a las que poder recurrir para una mejor comprensión e interpretación de los hechos sobre los que deberá informar (Quesada, 2007: 19). 
La condición de inesperado e imprevisible de buena parte de los hechos que protagonizan las páginas de sucesos debería limitar, como reflexiona la propia Quesada, la dependencia de las fuentes oficiales y de los comunicados de prensa. Nada más lejos de la realidad. Esta misma característica es la que lleva a los periodistas a depender en su trabajo exclusivamente de las fuentes oficiales (Quesada, 2003: 239).

La segunda variable más común en esta categoría es la que agrupa a las unidades en las que no consta la fuente de la que procede la información recogida. Su número asciende a 514 , que representa cerca de un $30 \%$ del conjunto de las unidades, lo que muestra la relevancia en la producción periodística analizada de una costumbre poco aconsejable, según la profesora Carmen Herrero.

Muchos de los problemas de la información de sucesos y de tribunales se derivan de la imposibilidad de citar explícitamente la fuente utilizada por el periodista, bien porque ésta no quiere, bien porque es un funcionario, bien porque es una fuente anónima, bien porque es inventada (Herrero, 2003: 113).

Como señala Núñez Ladevéze, siempre que sea posible, las fuentes han de ser identificadas claramente en el texto informativo (Núñez Ladevéze, 1995: 53).

El número de unidades disminuye de forma vertiginosa en las variables que recogen una descripción con mayor riqueza y variedad de fuentes. Así, aquéllas que incluyen fuentes institucionales, no institucionales y observación directa del redactor no alcanzan el $2 \%$.

\subsubsection{Origen de las fuentes por sucesos}

Las tendencias en la distribución de las fuentes informativas en función de su origen se mantienen también, en líneas generales, en los once sucesos objeto de estudio. Así, el grueso de las unidades redaccionales en la mayoría de los hechos analizados se surte de fuentes institucionales. Entre el primer suceso, el accidente aéreo del aeropuerto de Los Rodeos (1977), y el último, el accidente del autobús escolar en la localidad soriana de Golmayo (2000), la proporción de fuentes institucionales asciende ligeramente, del $28 \%$ al $31 \%$.

El segundo gran grupo de unidades redaccionales según el origen de las fuentes informativas es el que no hace constar ninguna. A diferencia del anterior, en este caso se aprecia un ligero descenso en la proporción entre el primer suceso por orden cronológico y el último. En concreto, son casi cinco puntos menos los de la proporción de unidades sin fuente identificada en el accidente de autobús en 2000 (25\%) respecto a la cobertura del siniestro aéreo de 1977 $(29,5 \%)$.

Otro tanto ocurre con el uso de otros diarios como fuente informativa en las unidades redaccionales. Del $11 \%$ registrado en el primer suceso objeto de estudio, 
92 La pobreza de las fuentes en la cobertura de sucesos de impacto por los diarios de referencia en España

el siniestro aéreo en el aeropuerto de Los Rodeos en 1977, se pasa, 23 años después, al $1 \%$ del accidente del autobús escolar en Soria. De hecho, a partir del tercer suceso, la explosión del colegio Marcelino Ugalde de Ortuella (1980), las proporciones recogidas de utilización de diarios como fuente de información no pasan del $3 \%$.

\subsubsection{Origen de las fuentes en los diarios analizados}

En tres de los rotativos (Abc, El Periódico de Catalunya y El Mundo), las unidades redaccionales en las que no consta la fuente informativa son mayoría. En el caso de Abc, el 37\% de sus unidades se agrupa bajo la variable "No consta". En El Periódico el porcentaje es del 34\% y en El Mundo del 33\%.

En el resto, las fuentes institucionales son las predominantes. Sobresale La Vanguardia de Barcelona, diario en el que el $42 \%$ de sus unidades redaccionales se nutre exclusivamente de fuentes institucionales. A escasa distancia se encuentra El Correo, con un 39\%, y más alejado, El País.

La tercera variable más relevante en términos porcentuales, la que refleja el uso combinado de fuentes institucionales y no institucionales, registra variaciones considerables. Se pueden perfilar tres grupos: el primero, formado por El País y Diario 16, con una proporción que ronda el 18\%; el segundo, compuesto por El Periódico, que se acerca al 14\%; y un tercero, el que forman Abc, La Vanguardia, El Correo y El Mundo, que se asienta sobre el 9\%.

En el uso de fuentes no institucionales, el rotativo más destacado, al igual que en la variable anterior, es El País (14\%), seguido por El Periódico de Catalunya $(13 \%)$ y El Correo (12\%). El resto se hallan por debajo del 10\% (la cabecera con una menor proporción de esta variable es Diario 16 con un 7\%).

Los porcentajes en el uso de otros diarios como fuentes informativas oscilan entre el 5\% de El Correo Español y el 0,4\% de El País y el 0,7\% de El Mundo. Por el contrario, es El Correo el que menos cita a las agencias informativa como fuente en sus unidades redaccionales $(2 \%)$, casi cuatro veces menos que la proporción de El Mundo, la mayor en esta variable, con un 9\%.

\subsection{Número de fuentes}

A tenor de los datos obtenidos, una de cada tres de las 1.750 unidades redaccionales analizadas incluye tres o más fuentes, en concreto, 552 , el $32 \%$ del total. Sin embargo, en una proporción similar, 512 unidades (el 29\% del conjunto del análisis) no reflejan fuente alguna en su texto. La tercera variable más numerosa es la que corresponde a una única fuente, que se da en una de cada cuatro unidades (432, el $25 \%$ ). Esto significa que más de la mitad de las unidades redaccionales analizadas o no tienen fuente alguna, o únicamente citan una, datos que justifican las palabras de Diezhandino (2007:148) cuando indica 
que la información de una sola fuente ya no es anatema, y a veces es una gran virtud "frente al olvido bastante generalizado de acudir y citar a las fuentes". Por último, el número de unidades con dos fuentes informativas es de 254 , el $15 \%$.

\subsubsection{Distribución del número de fuentes por sucesos}

El análisis por sucesos de los datos acerca del número de fuentes muestra modificaciones relevantes. En el caso de las unidades redaccionales con una sola fuente, se observa una clara tendencia decreciente en los ocho primeros sucesos, en los que la proporción baja del $37 \%$ del segundo suceso, la explosión en el camping tarraconense Los Alfaques (1978), hasta el 14\% registrado en la cobertura de los crímenes de Alcàsser (1992). Sin embargo, tras este suceso se produce un repunte que sitúa a las unidades redaccionales de los tres siguientes sucesos con una sola fuente informativa en porcentajes cercanos al $20 \%$, inferiores de cualquier modo a los constatados en los primeros.

Una evolución contraria se observa en las unidades con tres o más fuentes informativas. De hecho, la proporción que se obtiene en la cobertura del primer suceso (1977) es de un $26 \%$, y la del último un $40 \%, 13$ puntos más. El salto se sitúa a finales de los años 80 y principios de los 90 .

Una mayor estabilidad marca a las variables de unidades con ninguna fuente (entre el $25 \%$ y el $35 \%$ ) o con dos fuentes (alrededor del $15 \%$ ).

Cuadro 1. Distribución porcentual de las unidades redaccionales según el número de fuentes en cada suceso.

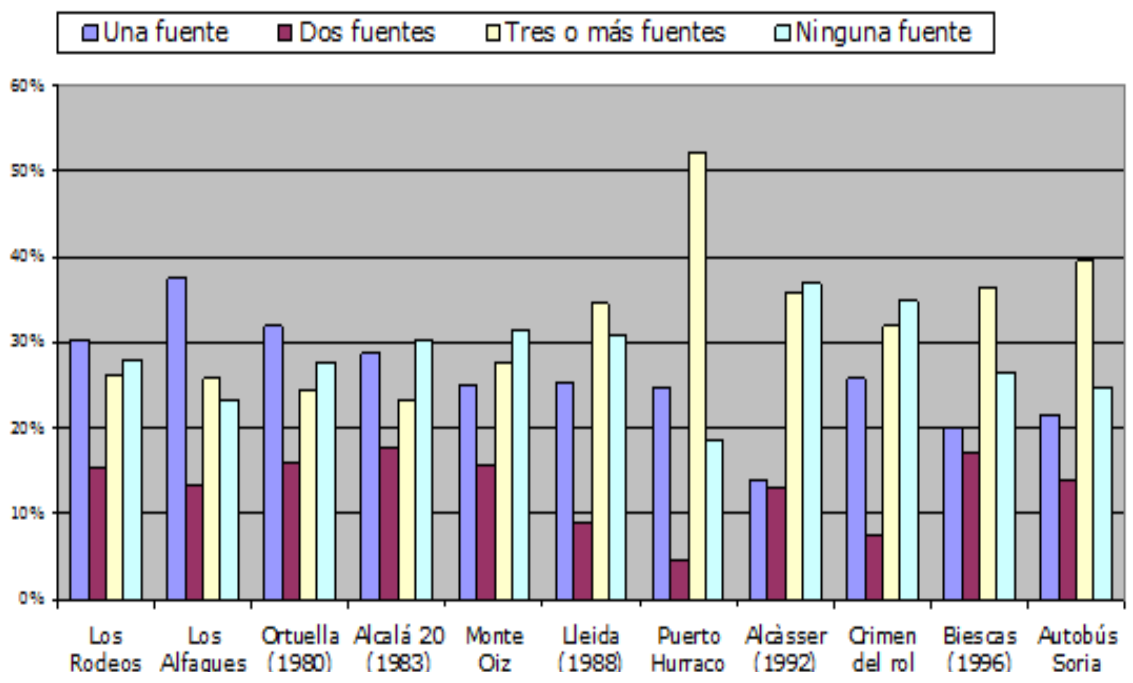

Fuente: Elaboración propia 
94 La pobreza de las fuentes en la cobertura de sucesos de impacto por los diarios de referencia en España

\subsubsection{Distribución del número de fuentes en los diarios analizados}

La observación del número de fuentes en los diarios objeto de estudio permite constatar algunos rasgos relevantes de las distintas cabeceras. En la variable que más se identifica con el periodismo de calidad, esto es, la que agrupa a las unidades redaccionales con tres o más fuentes informativas, destaca el diario El País, en el que el $39 \%$ de sus unidades cumple esa condición. Le siguen El Periódico de Catalunya, con un $37 \%$ y el diario El Mundo, con un $36 \%$. Con un $33 \%$ se sitúan La Vanguardia y Diario 16; y los rotativos con menor proporción en esta variable son El Correo Español, con un $27 \%$, y Abc, con un $23 \%$.

El orden se invierte si se toma como referencia la variable que recoge las unidades sin fuentes informativas. En este campo, el primer diario es Abc, con un $37 \%$ de sus unidades redaccionales sin fuente informativa citada, y el último es El País, con un $23 \%$. Entre uno y otro se sitúan, de más a menos, El Periódico de Catalunya (34\%), El Mundo (33\%), El Correo Español (27\%), La Vanguardia $(26 \%)$ y Diario $16(25 \%)$.

En el caso de las unidades redaccionales con una única fuente informativa, la mayor proporción se halla en El Correo Español, con un $32 \%$, al que le sigue La Vanguardia, con un $30 \%$ y Diario 16 , con un $26 \%$. El menor porcentaje se encuentra en los diarios El Mundo, 16\%, y El Periódico de Catalunya (15\%).

Cuadro 2. Distribución porcentual de las unidades redaccionales según el número de fuentes en cada diario.

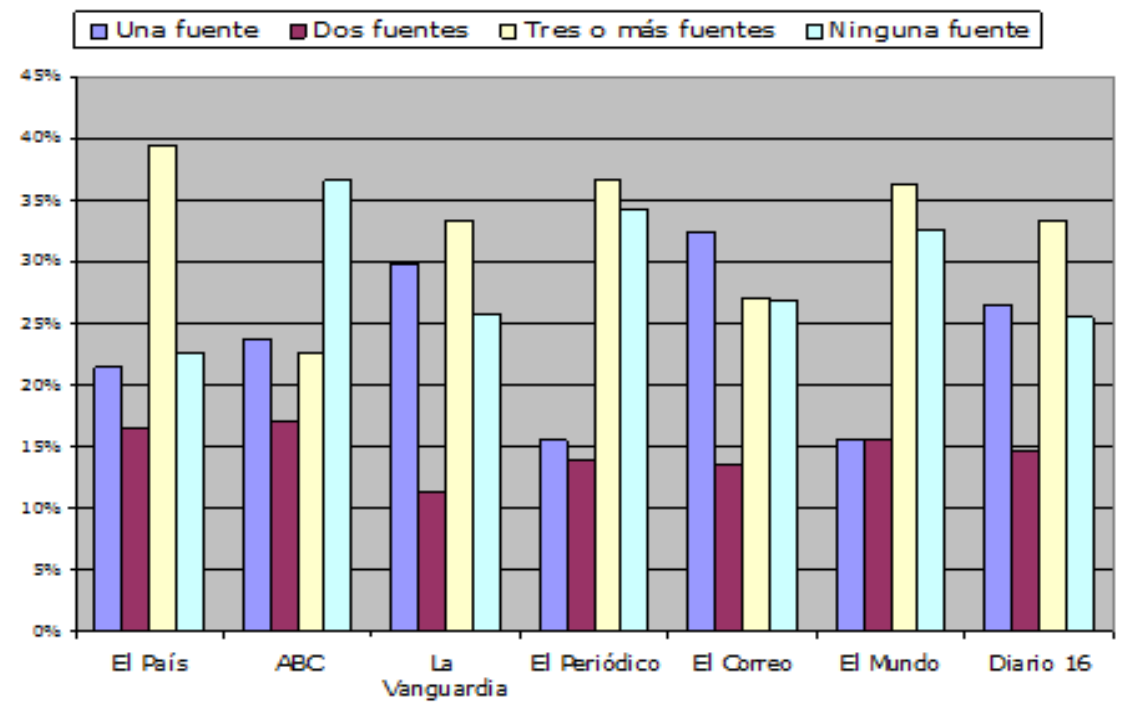

Fuente: Elaboración propia

\subsection{Declaraciones de testigos}

El uso de declaraciones de testigos en los sucesos no es una práctica muy 
extendida. En 1.179 unidades de las 1.750 analizadas, es decir, en el $67 \%$ del total, no se incluye declaración alguna de testigos.

\subsubsection{El uso de las declaraciones de testigos en cada suceso}

La utilización de las declaraciones de testigos crece de forma considerable en los sucesos que se ubican en la segunda mitad del periodo estudiado, en especial si se compara con los registros obtenidos en la primera etapa de la investigación. Así, se observa que en los cinco primeros sucesos, acaecidos entre 1977 y 1985, las proporciones de unidades redaccionales sin declaraciones de testigos se mueven en unos márgenes muy altos, entre el $75 \%$ y el $83 \%$. Sin embargo, estos porcentajes decrecen, y de forma considerable, en la cobertura de los sucesos ocurridos en la década de los 90. De hecho, las proporciones se mueven entre el 35\% (crímenes de Puerto Hurraco, 1990) y el 66\% (crímenes de Alcàsser, 1992).

Este dato representa la constatación de la tendencia creciente a una mayor utilización de las declaraciones de testigos en las unidades redaccionales. Se da mayor importancia al valor testimonial de este tipo de información y, por ello, aparece con mayor frecuencia. La mayor relevancia a esta aportación informativa en las unidades estudiadas se comprueba asimismo en el notable aumento de la variable que engloba las unidades que cuentan con mayor número de declaraciones de testigos, tres o más, que crece en una proporción muy superior a las de las variables de la declaración de un testigo o a las declaraciones de dos testigos. Como indican Israel y Pou, en el contexto actual la fuente no es solo el origen de la información y el aval de su fiabilidad sino que se convierte en ocasiones en el eje del relato. Deja de ser una herramienta del periodista, el canal por el que le llegan los datos de actualidad, para convertirse en el propio contenido. Lo novedoso no es la presencia del testimonio, sino su protagonismo en relación a las fuentes tradicionales (Israel y Pou, 2006: 171). En los primeros sucesos, los cinco que se sitúan entre 1977 y 1985, las proporciones de unidades que incluyen declaraciones de tres o más testigos se mueven en márgenes muy pequeños, entre el $1 \%$ (Los Alfaques, 1978) y el $6 \%$ (incendio de la discoteca Alcalá 20 en 1983). Esta situación cambia en los últimos cinco sucesos, entre 1990 y 2000, en los que las proporciones de unidades que incluyen declaraciones de tres o más testigos van del $13 \%$ (Crímenes de Alcàsser en 1992) al $25 \%$ (accidente de un autobús escolar en Soria, 2000). 
96 La pobreza de las fuentes en la cobertura de sucesos de impacto por los diarios de referencia en España

Cuadro 3. Distribución porcentual de las unidades redaccionales en función del uso de declaraciones de testigos en cada suceso.

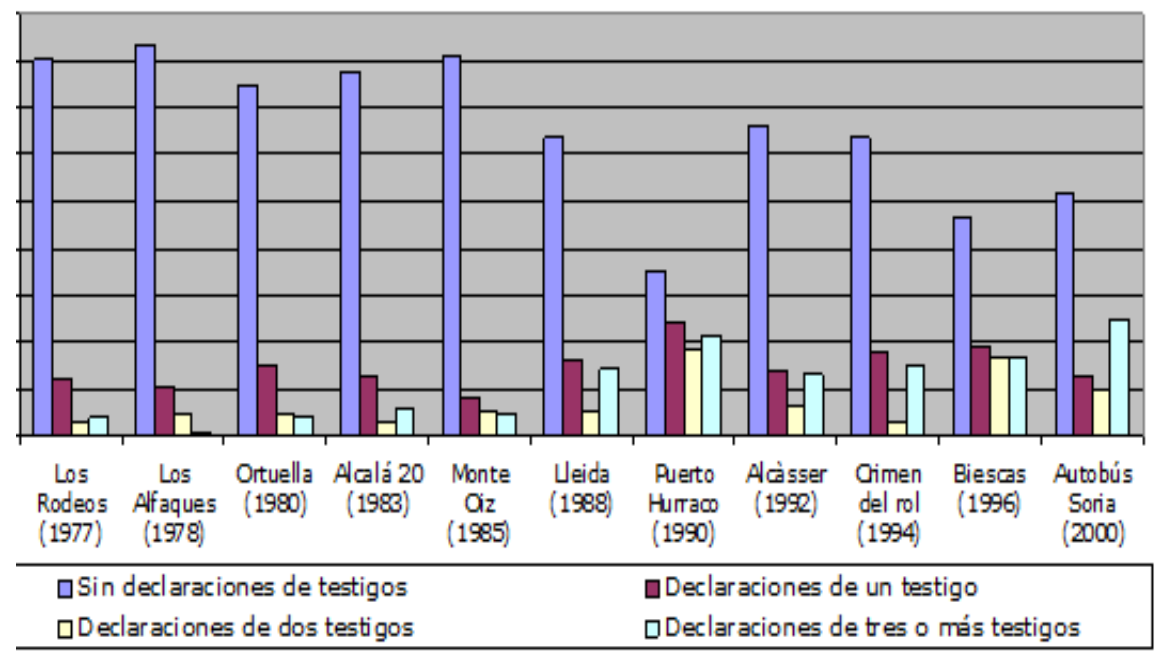

Fuente: Elaboración propia

\subsubsection{El uso de declaraciones de testigos en cada uno de los diarios}

El rotativo que menos se decanta por las declaraciones de testigos en sus artículos, informaciones o reportajes es Abc, ya que el $77 \%$ de sus 328 unidades estudiadas, es decir, 252, no incluyen ningún testimonio de testigos. En una línea similar se sitúa El Correo Español, con un $71 \%$ de sus 383 unidades sin declaraciones de testigos. En el lado contrario, destacan El Mundo y El Periódico de Catalunya, en los que la proporción de unidades redaccionales sin declaraciones de testigos se limita al $56 \%$ y al $58 \%$, lo que supone que el $44 \%$ y el $42 \%$ de sus unidades sí utilizan, al menos, la declaración de un testigo. Por su parte, El País, La Vanguardia y Diario 16 están en porcentajes muy cercanos al $67 \%$ de la media general del estudio en este aspecto.

\subsection{Declaraciones de afectados y de familiares}

La utilización de los afectados de forma directa o indirecta como fuente en la cobertura de los sucesos estudiados es muy reducida. Casi el $87 \%$ de las 1.750 unidades redaccionales estudiadas no incluyen ninguna declaración de estas personas. En el 13\% restante, 231 unidades, predomina el uso del testimonio de un afectado o familiar: se da en 134 unidades, cerca del $8 \%$ del total. La suma de las unidades que cuentan con dos testimonios de afectados o familiares (47) o de tres o más (50) no alcanza el $6 \%$ del conjunto del estudio. 


\subsubsection{El uso de declaraciones de afectados y de familiares en cada} suceso y en cada diario

La reducida utilización de declaraciones de afectados y de familiares es una constante en la cobertura de los sucesos analizados. No obstante, se comprueba una ligera tendencia creciente. Si se observa el uso de declaraciones de afectados y familiares en cada uno de los diarios estudiados, se comprueba que hay diferencias sensibles. En Abc solo el $9 \%$ de sus unidades incluye testimonios de afectados y/o familiares. En porcentajes similares se sitúan El Correo Español $(10 \%)$, El País $(11,5 \%)$ y La Vanguardia $(12,2 \%)$. Por el contrario, en El Mundo el $22 \%$ de sus unidades incluye testimonios de este tipo, proporción que se acerca al $20 \%$ en Diario 16 y El Periódico de Catalunya.

\section{CONCLUSIONES}

La pobreza de fuentes caracteriza la cobertura periodística de los diarios de referencia españoles en sucesos de impacto. Pobreza en dos sentidos, el cuantitativo y el cualitativo. Pobreza cuantitativa porque se utilizan y se citan pocas fuentes. Tanto es así que más de la mitad, un $53 \%$, de las unidades redaccionales analizadas no citan fuente alguna, o se contentan con incluir solo una. La pobreza se mantiene en un plano cualitativo. Una de cada tres de las 1.750 unidades estudiadas se basa de manera exclusiva en fuentes institucionales. El número de unidades disminuye de forma vertiginosa en las variables que recogen una descripción con mayor riqueza y variedad de fuentes.

No obstante, se aprecia una evolución positiva en el uso, al menos desde una perspectiva cuantitativa, de fuentes informativas, materializada en la transformación de la variable que se identifica en mayor medida con el periodismo de calidad, esto es, la que agrupa a las unidades redaccionales con tres o más fuentes informativas. La proporción que se obtiene en la cobertura del primer suceso (1977) es de un $26 \%$, y la del último un $40 \%, 13$ puntos más. El salto se sitúa a finales de los años 80 y principios de los 90 . Pese a todo, la proporción de unidades sin fuente alguna se mantiene en el $25 \%$, al igual que las unidades que recurren a una única fuente.

La pobreza generalizada en el uso de las fuentes registra matices notables en cada uno de los diarios analizados. La cabecera que sale mejor parada es El País, tanto en términos cuantitativos como cualitativos. Cuatro de cada diez de sus unidades redaccionales incluyen tres o más fuentes informativas; es el periódico que obtiene mayores proporciones de uso de fuentes no institucionales; el que menos recurre a agencias o a datos de otros diarios y el que menor proporción de noticias sin fuentes citadas recoge. En el siguiente peldaño se sitúan La Vanguardia, El Mundo, El Periódico de Catalunya y Diario 16. Los peores datos corresponden a El Correo y a Abc. En este último, solo un $23 \%$ de las unidades redaccionales cuenta con tres o más fuentes, mientras que el $37 \%$ no tiene ninguna fuente. 
98 La pobreza de las fuentes en la cobertura de sucesos de impacto por los diarios de referencia en España

El carácter testimonial de la cobertura periodística de los sucesos aumenta de forma considerable a lo largo del periodo estudiado. En números totales, una de cada tres unidades redaccionales analizadas cuenta con declaraciones de testigos, pero las diferencias son considerables desde una perspectiva diacrónica. Así, en los cinco primeros sucesos, acaecidos entre 1977 y 1985, las proporciones de unidades redaccionales con declaraciones de testigos se mueven en unos márgenes muy bajos, entre el $17 \%$ y el $25 \%$. Sin embargo, estos porcentajes aumentan, y de forma considerable, en la cobertura de los sucesos ocurridos en la década de los 90. De hecho, las proporciones se mueven entre el 34\% (crímenes de Alcàsser, 1992) y 65\% (crímenes de Puerto Hurraco, 1990).

Otro dato que corrobora el auge del periodismo de testimonios es el incremento de las unidades redaccionales con tres o más testimonios, cuyo porcentaje en los últimos sucesos (entre el $13 \%$ y el $25 \%$ ) está muy por encima del registrado en los primeros (no pasa del 6\%).

El énfasis en la apuesta por el carácter testimonial y emotivo de la cobertura periodística varía en función del diario analizado. A tenor de los resultados, los rotativos que más han apostado por esa vía son El Mundo y El Periódico de Catalunya. En el caso del diario madrileño, el $44 \%$ de sus unidades redaccionales cuentan con, al menos, la declaración de un testigo y el $22 \%$ incluye uno o más declaraciones de afectados o de familiares, números ligeramente superiores a los de El Periódico de Catalunya (42\% y 20\% respectivamente). Estos datos casi doblan a los que se contabilizan en Abc y El Correo, los diarios que menor proporción de testimonios recogen.

\section{REFERENCIAS BIBLIOGRÁFICAS}

ARMENTIA J.I./ CAMINOS J.M. (1998): La información. Redacción y estructuras. Bilbao: Universidad del País Vasco.

ARMENTIA, J.I y MARÍN, F. La extinción de la prensa. En VV AA (2009): Estudios de Periodística XV. El drama del periodismo: Narración e información en la cultura del espectáculo. Murcia: Editado por la Sociedad Española de Periodística en el Servicio de Publicaciones de la UCAM. Pp. 521-531.

BEZUNARTEA, O. DEL HOYO M. y MARTÍNEZ F. (1998): 21 lecciones de reporterismo. Zarauz: Universidad del País Vasco.

BORRAT, H.: Las relaciones noticiables: fuentes-autores. En LOSADA VÁZQUEZ, A.; ESTEVE RAMÍREZ, F. (eds) (2003): El periodismo de fuente. Salamanca: Publicaciones de la Universidad Pontificia de Salamanca. Pp. 67-85.

CASALS CARRO, M. J. (2005): Periodismo y sentido de la realidad / Teoría y análisis de la narrativa periodística. Madrid: Fragua.

CHIMENO RABANILLO, S.: Las fuentes, en el proceso de la información periodística especializada. En ESTEVE RAMÍREZ, E. (Coord.) (1997): Estudios sobre Información Periodística Especializada. Valencia: Fundación Universitaria CEU San Pablo. Pp 43-61. 
DIEZHANDINO, M.P. y COCA, C. (1997). La nueva información. Bilbao: Servicio editorial de la UPV.

DIEZHANDINO, P. (2007): Periodismo y poder. Madrid: Pearson Educación.

FUENTES, J.F. y FERNÁNDEZ SEBASTIÁN, J. (1998). Historia del periodismo español. Madrid: Editorial Síntesis.

GANS, H.J. (1980): Deciding what's news. A study of CBS Evening News, NBC Nightly News, Newsweek and Time. New York: Vintage Book Editions.

GARCÍA SANTAMARÍA, J.V. (2010): Crisis del periodismo de fuentes. Las prácticas del periodismo en España en el accidente de Spanair. Revista Latina de Comunicación Social [en línea], 65. La Laguna (Tenerife): Universidad de La Laguna, páginas 516 a 537 [Consulta: 5 de julio de 2011].

http://www.revistalatinacs.org/10/art3/916_UC3M/38_Santamaria.html

GIL CALVO, E., ORTIZ, J. y REVUELTA, M. (2002): Repensar la prensa. Madrid: Editorial Debate.

HERRERO AGUADO C. Y GARCÍA GORDILLO M.: Periodismo de fuente: de la información política a la de sucesos. En LOSADA VÁZQUEZ, A.; ESTEVE RAMÍREZ, F. (eds) (2003): El periodismo de fuente. Salamanca: Publicaciones de la Universidad Pontificia de Salamanca. Pp. 241-259.

HERRERO, C. (2003). Periodismo de sucesos y tribunales. Tratamiento de la violencia social. Serie Comunicación. Sevilla: Padilla Libros Editores y Libreros.

ISRAEL GARZÓN, E. Y POU AMÉRICO, M.J. La personalización de las fuentes. Voces de la sociedad entre el testimonio y la denuncia. En VV AA (2006): Estudios de Periodística XII. Fuentes informativas: Sigilo y Transparencia. Facultad de Humanidades y Ciencias de la Comunicación. Universidad San Pablo CEU. Madrid.

LARRAÑAGA, J. "La demanda de los periódicos impresos y online: un análisis económico". Ámbitos. Revista Internacional de Comunicación, número 18 (2009). Pp 23-39.

LÓPEZ, M. (1995): Cómo se fabrican las noticias. Fuentes, selección y planificación. Barcelona: Paidós.

LÓPEZ, X. (2006). Periodistas-fuentes: focos de tensión de una relación siempre compleja. En VV AA (2006): Estudios de Periodística XII. Fuentes informativas: Sigilo y Transparencia. Facultad de Humanidades y Ciencias de la Comunicación. Madrid:

Universidad San Pablo CEU.

LÓPEZ, X. y GARCíA, B. (2005): Radiografía dos diarios Galegos. Análise dos contenidos textuais e dos aspectos formais. Santiago de Compostela: Servicio Editorial de la Universidad de Santiago de Compostela.

MORENO SARDÁ, A. (1998): La mirada informativa. Barcelona: Editorial Bosch.

NÚÑEZ LADEVÉZE, J.L. (1995): Introducción al periodismo escrito. Barcelona: Ariel.

PABLOS COELLO, J.M. : "Fuentes mudas (en la web): periodismo transitpropaganda". Estudios sobre el Mensaje Periodístico, número 12 (2006). Pp 115-144.

PIEDRAHITA, M. (1993): Periodismo moderno. Historia, perspectivas y tendencias hacia 
100a pobreza de las fuentes en la cobertura de sucesos de impacto por los diarios de referencia en España

el año 2000. Madrid: Editorial Paraninfo.

QUESADA, M. (2007): Periodismo de sucesos. Madrid: Editorial Síntesis.

QUESADA, M.: La especialización en sucesos y tribunales. En LOSADA VÁZQUEZ, A.; ESTEVE RAMÍREZ, F. (eds) (2003): El periodismo de fuente. Salamanca: Publicaciones de la Universidad Pontificia de Salamanca. Pp. 233-241.

SINGER, E., and ENDRENY, P.M. (1993): Reporting on risk. How the Mass Media Portray Accidents, Diseases, Disasters, and other Hazards. New York: Rusell Sage Foundation.

STRENTZ, H. (1983): Periodistas y fuentes informativas. Buenos Aires: Marymar.

VV. AA (2002). El diario de servicios en España. Oviedo: Septem Ediciones.

\section{Breve semblanza de los autores}

Iñigo Marauri Castillo (Vitoria, 1973) es profesor del Departamento de Periodismo II de la Universidad del País Vasco. Doctor en Periodismo, ha trabajado como redactor en los diarios El Correo y El País. Ha sido director adjunto y responsable de investigaciones de la revista de información al consumidor Consumer Eroski (www.consumer.es), así como subdirector del proyecto multimedia Consumidores de la radio televisión pública vasca (EITB).

María del Mar Rodríguez González (San Sebastián, 1974) es doctora en Periodismo por la Universidad del País Vasco. Especialista en comunicación de crisis y en periodismo de servicio en la web, ejerce como responsable de contenidos de la publicación de información al consumidor Consumer Eroski, tanto en su versión impresa como en su versión online (www.consumer.es).

María José Cantalapiedra González (Barakaldo, 1969) es profesora titular del Departamento de Periodismo II de la Universidad del País Vasco. Especialista en periodismo digital, ha dirigido varias tesis doctorales y ha publicado artículos en revistas científicas nacionales e internacionales. 